\title{
Osteocondroma retroescapular como diagnóstico diferencial de escápula alada*
}

\section{Subscapular Osteochondroma as a Differencial Diagnosis of Winging of Scapula}

\author{
Marco Aurélio de Oliveira ${ }^{1}$ Yuri Alfaro ${ }^{1}$ Anastácio Kotzias Neto $^{1}$ Mário César Korman ${ }^{1}$ \\ ${ }^{1}$ Hospital Infantil Joana de Gusmão, Florianópolis, SC, Brasil \\ Address for correspondence Marco Aurélio de Oliveira, Hospital \\ Infantil Joana de Gusmão, Rua Rui Barbosa, 152, Agronômica, \\ Rev Bras Ortop 2019;54:241-246. \\ Florianópolis, SC, 88025-301, Brazil \\ (e-mail: marco.oliveirasc@yahoo.com.br).
}

\section{Resumo \\ Palavras-Chave \\ - osteocondroma \\ - escápula \\ - neoplasias ósseas}

\section{Abstract}

Objetivo O objetivo do presente trabalho é relatar as características clínicas de pacientes pediátricos com diagnóstico de osteocondroma retroescapular submetidos a tratamento cirúrgico, entre os anos de 2003 e 2017.

Métodos Série de casos, analítica, descritiva e retrospectiva de sete pacientes com diagnóstico de osteocondroma retroescapular.

Resultados A média de idade dos pacientes analisados foi de 9,5 anos, sendo $71 \%$ deles do sexo masculino. $O$ tempo médio entre o início dos sintomas e o procedimento cirúrgico foi de 1,2 anos. Aproximadamente $71 \%$ dos pacientes apresentaram osteocondroma na escápula direita, e $57,1 \%$ dos casos foram classificados como sésseis. Ao exame clínico, observou-se pseudoescápula alada em $85,7 \%$, crepitação em $71,4 \%$, e queixa de dor em $42,9 \%$ dos pacientes.

Conclusão A escápula alada pode ter diferentes etiologias, dentre elas o osteocondroma retroescapular. O conhecimento sobre anatomia funcional e semiologia ortopédica somado à correta sistematização da abordagem dos tumores ósseos consiste na base para o correto diagnóstico diferencial e tratamento adequado.

Objective The aim of the present study is to report the clinical characteristics of pediatric patients with a diagnosis of retroescapular osteochondroma submitted to surgical treatment at the Hospital Infantil Joana de Gusmão (HIJG) in Florianópolis, state of Santa Catarina, Brazil, between 2003 and 2017.

Methods Analytical, descriptive and retrospective case series of seven patients with the diagnosis of retroescapular osteochondroma.

Results The mean age of the patients analyzed was 9.5 years, with $71 \%$ of them being male. The mean time between the onset of symptoms and the surgical procedure was of 1.2 years. Approximately $71 \%$ of the patients presented osteochondroma of the right scapula, and $57.1 \%$ of the cases were classified as sessile. At the clinical examination, winged scapula was observed in $85.7 \%$, crepitus in $71.4 \%$, and pain complaint in $42.9 \%$ of the patients.

Trabalho feito no Hospital Infantil Joana de Gusmão, Florianópolis, SC, Brasil.

(1) Yuri Alfaro's ORCID is https://orcid.org/0000-0003-1793-1971.

received

February 24, 2018

accepted

July 24, 2018
DOI https://doi.org/

10.1055/s-0039-1692432. ISSN 0102-3616.
Copyright $\odot 2019$ by Sociedade Brasileira License terms de Ortopedia e Traumatologia. Published by Thieme Revnter Publicações Ltda, Rio de Janeiro, Brazil 


\section{Keywords}

- osteochondroma

- scapula

- bone neoplasms
Conclusion Winged scapula can have different etiologies, among them the retroescapular osteochondroma. The knowledge about functional anatomy and orthopedic semiology added to the correct systematization of the approach of bone tumors is the basis for the correct differential diagnosis and adequate treatment.

\section{Introdução}

O osteocondroma é uma exostose óssea, com projeção da cortical contínua com o osso subjacente, preenchida por osso esponjoso e coberta por tecido cartilaginoso que possui espessamento de entre 1 e $3 \mathrm{~mm}$, provavelmente originado da modificação de crescimento da placa fisária, que acompanha o ritmo de crescimento, levando ao aparecimento de proeminências ósseas. ${ }^{1}$

O osteocondroma corresponde a entre 10 e $15 \%$ de todos os tumores ósseos. Dentre os benignos, é o mais comum, representando entre 30 e $50 \%$ dos casos. ${ }^{2,3}$ Em sua maioria, são lesões únicas, podendo se apresentar de duas formas: séssil ou pediculada. Acometem principalmente a região metafisária de ossos longos, em especial o joelho (fêmur distal e tíbia proximal) e o úmero proximal, acometendo raramente os ossos planos. Entretanto, é o tumor benigno mais comum da escápula, correspondendo a $\sim 5 \%$ dos $\operatorname{casos}^{2,3}$ (-Fig. 1).

Mais comum em pacientes com idade $<30$ anos, sua prevalência é maior no sexo masculino, com uma relação de $1,5 .{ }^{4,5}$ É rara sua malignização, ocorrendo em $\sim 2 \%$ dos casos quando na forma solitária. 6,7

A escápula alada é uma síndrome com diversas etiologias, sendo a causa mais comum relacionada à lesão do nervo torácico longo. Porém, o osteocondroma retroescapular é um importante diagnóstico diferencial e, neste caso, a denominação correta torna-se pseudoescápula alada, caracterizando-se por uma deformidade escapular móvel ou fixa., Descrita por McWilliams em 1914, a pseudoescápula alada secundária ao osteocondroma é de difícil diferenciação com a escápula alada decorrente da lesão do nervo torácico longo e deverá estar sempre dentre as possibilidades diagnósticas para este tipo de manifestação clínica. ${ }^{10,11}$

Na literatura, poucos são os trabalhos que relacionam a escápula alada ao osteocondroma retroescapular, sendo em sua maioria relatos de casos.

Diante disso, o objetivo do presente trabalho é relatar as características clínicas de pacientes pediátricos com diagnóstico de osteocondroma retroescapular submetidos a tratamento cirúrgico no Hospital Infantil Joana de Gusmão (HIJG) em Florianópolis, SC, Brasil.

O presente estudo foi aprovado de Comitê de Ética e Pesquisa do HIJG sob o número 2.213.465.

\section{Materiais e Métodos}

O presente trabalho é considerado uma série de casos analítica, descritiva e retrospectiva.

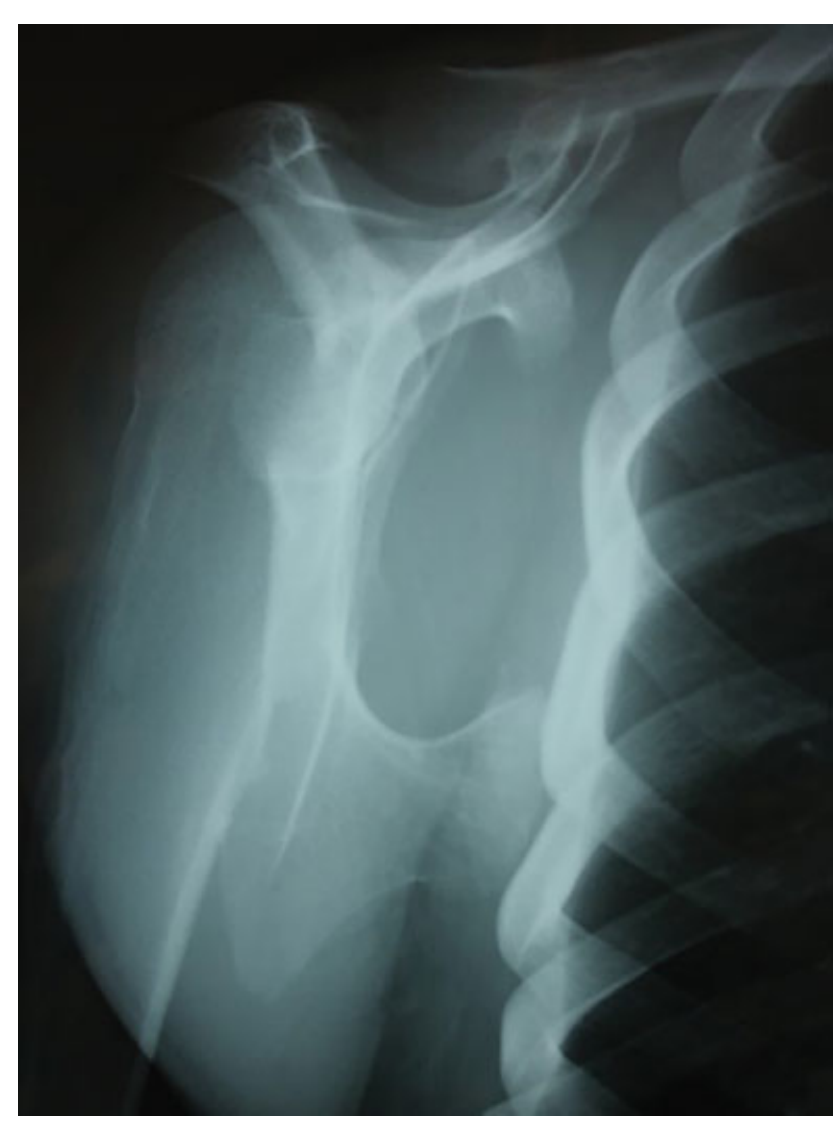

Fig. 1 Radiografia axial da escápula apresentando o osteocondroma no terço médio do seu aspecto anterior.

Foram avaliados 7 prontuários de indivíduos com idades de 4 a 14 anos submetidos ao tratamento cirúrgico de ressecção de osteocondroma retroescapular no Hospital Infantil no serviço de Ortopedia e Traumatologia, do período de fevereiro de 2003 a março de 2017.

0 instrumento para coleta dos dados foi criado pelos próprios autores, através de um protocolo desenvolvido para o presente estudo, que aborda questões sobre os dados socioeconômicos, faixa etária, sexo, localização das lesões, análise descritiva das características tumorais e clínicas dos pacientes estudados.

As informações coletadas foram armazenadas em banco de dados em meio eletrônico, no software Microsoft Excel (Microsoft Corporation, Redmond, WA, EUA). As variáveis categóricas serão descritas por meio de sua frequência absoluta ( $n$ ) e relativas (\%). A análise estatística foi realizada com uso do software estatístico SPSS Statistics for Windows, Versão 22.0 (IBM Corp., Armonk, NY, EUA). 


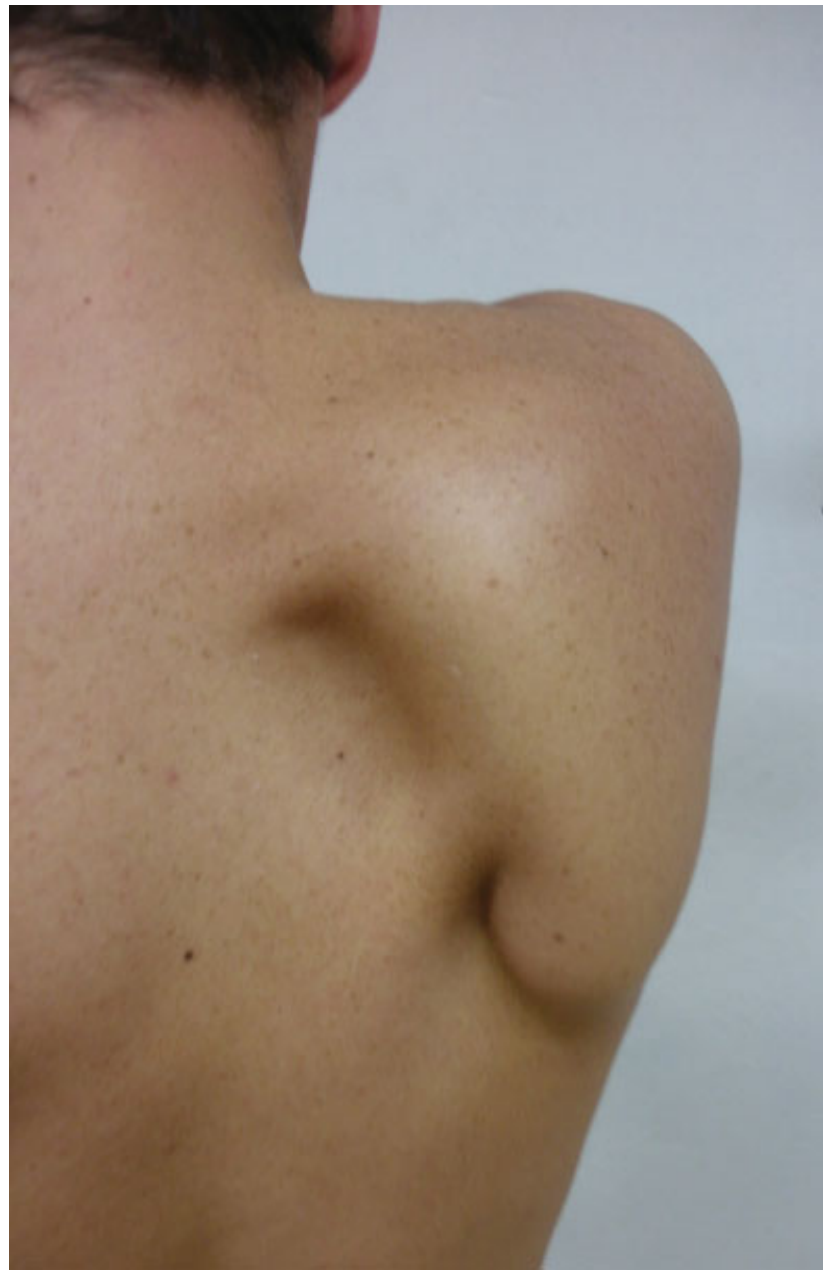

Fig. 2 Imagem clínica do osteocondroma retroescapular.

\section{Resultados}

Foram avaliados sete prontuários de pacientes com diagnóstico de osteocondroma retroescapular e submetidos ao tratamento cirúrgico no HIJG entre os anos de 2003 e 2017.

Dos pacientes estudados, $71 \%$ pertenciam ao sexo masculino. A média de idade foi de 9 anos e 5 meses, com idades variando entre 4 e 14 anos. O tempo médio de início dos sintomas e o procedimento cirúrgico foi de 1 ano e 2 meses.
Quanto às características relacionadas ao tumor, 5 pacientes $(71,4 \%)$ apresentavam osteocondroma de escápula direita, e $2(28,6 \%)$ à esquerda. Deste total, 57,1\% eram sésseis, e $\sim 43 \%$ dos casos apresentavam o tumor na região escapular (solitário) (-Fig. 2).

A respeito do quadro clínico, a maioria dos pacientes (85,7\%) apresentava pseudoescápula alada ao exame físico, e $71,4 \%$ apresentavam crepitação. Menos da metade dos pacientes $(42,9 \%)$ se queixavam de dor. Bursite esteve presente em três dos sete pacientes estudados (-Tabela 1).

Quanto às distribuições de casos, entre as variáveis de observação, pode-se perceber que há predominância do lado direito, "alada" (snapping) e ausência de bursite (seis casos em sete para todas essas variáveis). Já entre tipo, forma, dor e crepitação, verifica-se equilíbrio entre presença e ausência (-Tabela 2).

\section{Discussão}

O osteocondroma corresponde a entre 30 e 50\% dos tumores ósseos benignos e apenas $4 \%$ dos tumores escapulares são desta etiologia. A primeira descrição do osteocondroma com deformidade escapular coube a McWilliams em 1914, por meio de relato de caso. ${ }^{10}$

O osteocondroma é o tumor benigno mais comum na região metafisária dos ossos longos e raramente envolve ossos planos; entretanto, é o tumor benigno mais frequente na escápula. 2,3

A sua presença e, por consequência, seu diagnóstico, é observado com o crescimento e a presença do aumento aparente de volume na região escapular à ectoscopia. A manifestação clínica, como dor local, tende a ser mais tardia e pode ser secundária à compressão de estruturas neurovasculares, ao espessamento da bursa (quando presente), à fratura do pedículo ou à malignização. Outros sintomas, como ressalto escapular, crepitação, edema e perda da mobilidade, poderão estar presentes e dependerão do tamanho e da localização do tumor tanto nos planos sagital como coronal. ${ }^{12}$ A denominada "pseudoescápula alada" é observada quando o tumor se localiza na borda medial, inferior e ventral da escápula, onde ocorre deslocamento da concavidade do tumor sobre a face convexa do gradil costal, levando

Tabela 1 Base de dados

\begin{tabular}{|l|l|l|l|l|l|l|l|l|l|l|}
\hline Nome & Gênero & Idade & $\begin{array}{l}\text { Tempo de } \\
\text { evolução }\end{array}$ & Lado & $\begin{array}{l}\text { Solitário/ } \\
\text { Múltiplo }\end{array}$ & $\begin{array}{l}\text { Séssil/ } \\
\text { Pediculado }\end{array}$ & Dor & $\begin{array}{l}\text { Escápula } \\
\text { alada }\end{array}$ & Crepitação & Bursite \\
\hline 1 & M & 14 & 1.7 & Direito & Solitário & Pediculado & Não & Sim & Sim & Sim \\
\hline 2 & M & 14 & 0.9 & Direito & Múltiplo & Séssil & Sim & Sim & Sim & Não \\
\hline 3 & F & 6 & 1.3 & Esquerdo & Múltiplo & Séssil & Não & Sim & Sim & Não \\
\hline 4 & M & 4 & 1.2 & Direito & Múltiplo & Pediculado & Não & Sim & Não & Não \\
\hline 5 & M & 12 & 1.8 & Esquerdo & Solitário & Séssil & Sim & Sim & Sim & Sim \\
\hline 6 & F & 10 & 1.2 & Direito & Múltiplo & Séssil & Não & Sim & Sim & Não \\
\hline 7 & M & 7 & 0.7 & Direito & Solitário & Pediculado & Sim & Não & Não & Sim \\
\hline
\end{tabular}

Fonte: elaboração do autor, 2017. 
Tabela 2 Análise estatística

\begin{tabular}{|c|c|}
\hline Variável & Frequência (\%) \\
\hline \multicolumn{2}{|l|}{ Lado } \\
\hline Esquerdo & $1(14,3)$ \\
\hline Direito & $6(85,7)$ \\
\hline \multicolumn{2}{|l|}{ Tipo } \\
\hline Único & $3(42,9)$ \\
\hline Múltiplo & $4(57,1)$ \\
\hline \multicolumn{2}{|l|}{ Forma } \\
\hline Séssil & $3(42,9)$ \\
\hline Pediculado & $4(57,1)$ \\
\hline \multicolumn{2}{|l|}{ Dor } \\
\hline Presente & $3(42,9)$ \\
\hline Ausente & $4(57,1)$ \\
\hline \multicolumn{2}{|c|}{ “Alada” (Snapping) } \\
\hline Sim & $1(14,3)$ \\
\hline Não & $6(85,7)$ \\
\hline \multicolumn{2}{|l|}{ Crepitação } \\
\hline Sim & $4(57,1)$ \\
\hline Não & $3(42,9)$ \\
\hline \multicolumn{2}{|l|}{ Bursite } \\
\hline Sim & $1(14,3)$ \\
\hline Não & $6(85,7)$ \\
\hline
\end{tabular}

Fonte: Elaboração do autor, 2017.

a escápula para a região lateral da cintura escapular. Isto acontece quando o paciente faz abdução associada à rotação interna do ombro. ${ }^{13}$ Esta situação é muito semelhante à paralisia do nervo torácico longo, no qual realizamos a manobra para avaliar a integridade do músculo serrátil anterior, na qual o paciente empurra uma superfície longitudinal ao eixo dos membros superiores com os cotovelos estendidos. $O$ teste é considerado positivo quando há excesso de deslocamento lateral da escápula de maneira assimétrica com o lado oposto, configurando, assim, a "escápula alada" (-Fig. 3).

São também considerados diagnósticos diferencias para a escápula alada: neuralgia amiotrófica, lesão do nervo acessório espinhal, discinesias escapulotorácicas, e qualquer outra distrofia fascioescapulotorácica ${ }^{12,14}$ (- Fig. 4).

Fiddian et $\mathrm{al}^{15}$ classificaram a escápula alada quanto à mobilidade em estática (A) e dinâmica (B), e em quatro tipos quanto à origem anatômica da lesão: tipo I, causada por lesão nervosa (mais comum), tipo II, relacionada a lesão muscular, tipo III, por etiologia óssea, e tipo IV, por alteração dano articular. Todos os pacientes estudados foram classificados com escápula alada dinâmica de origem óssea (B-III)

A malignização do osteocondroma é incomum. Nas formas solitárias, ocorre em $\sim 2 \%$, e para as formas múltiplas, em $\sim 27 \%{ }^{16}$ A literatura enfatiza que a espessura da capa cartilaginosa é fator preditivo da agressividade, ou seja,

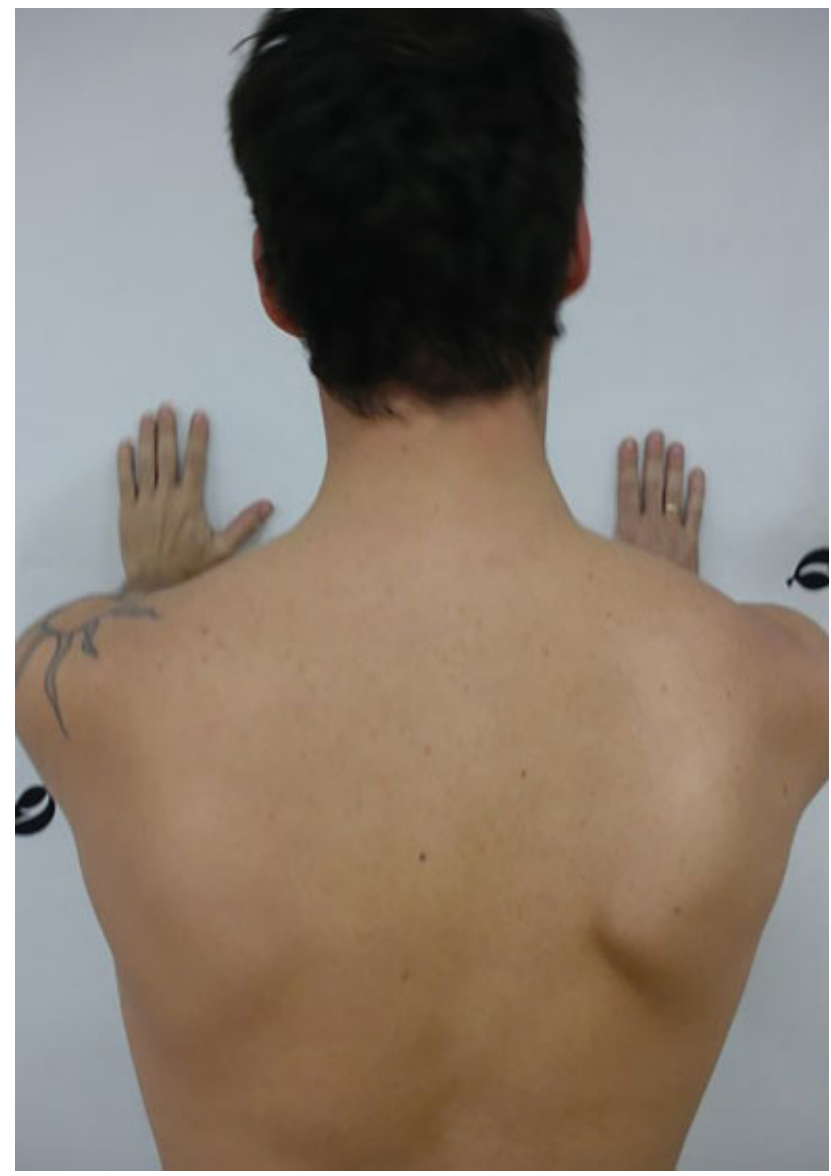

Fig. 3 Imagem clínica da escápula alada.

espessura $>2,0 \mathrm{~cm}$ é um forte indício de malignização, associada à formação de calcificações em sua margem. ${ }^{12,16}$ Quanto às manifestações clínicas, é preciso ficar atento ao crescimento do osteocondroma, após a maturidade esquelética, visto que estes tumores tendem a encerrar o seu crescimento durante a $2^{\underline{a}}$ década de vida. Em todos os nossos casos operados, o material ressecado foi encaminhado à anatomia patológica, sendo descartada a presença de células malignas.

A recorrência do osteocondroma pós-ressecção é de 2\% e deve-se à permanência de resíduos da cartilagem e de seu pericôndrio no ato cirúrgico. ${ }^{17}$ Não encontramos recorrências tumorais em nosso estudo.

Estudos radiográficos anteroposterior, perfil de escápula e axilar são suficientes para a identificação das lesões. Na dificuldade de interpretação, a tomografia computadorizada (TC) poderá ser útil para melhorar a estratégia quanto à via de acesso. Por se tratar de lesão cartilaginosa, a ressonância magnética (RM) é um excelente exame, principalmente no sentido de avaliar o espessamento da capa cartilaginosa e presença e extensão da bursa, quando presente.

Vários autores descrevem a presença de bursa entre o osteocondroma e as partes moles envolvidas (músculo grande dorsal e músculo serrátil anterior). Ela é formada pelo atrito do tumor com o gradil costal, formando líquido sinovial, sendo causa de dor nesta região devido à propensão 


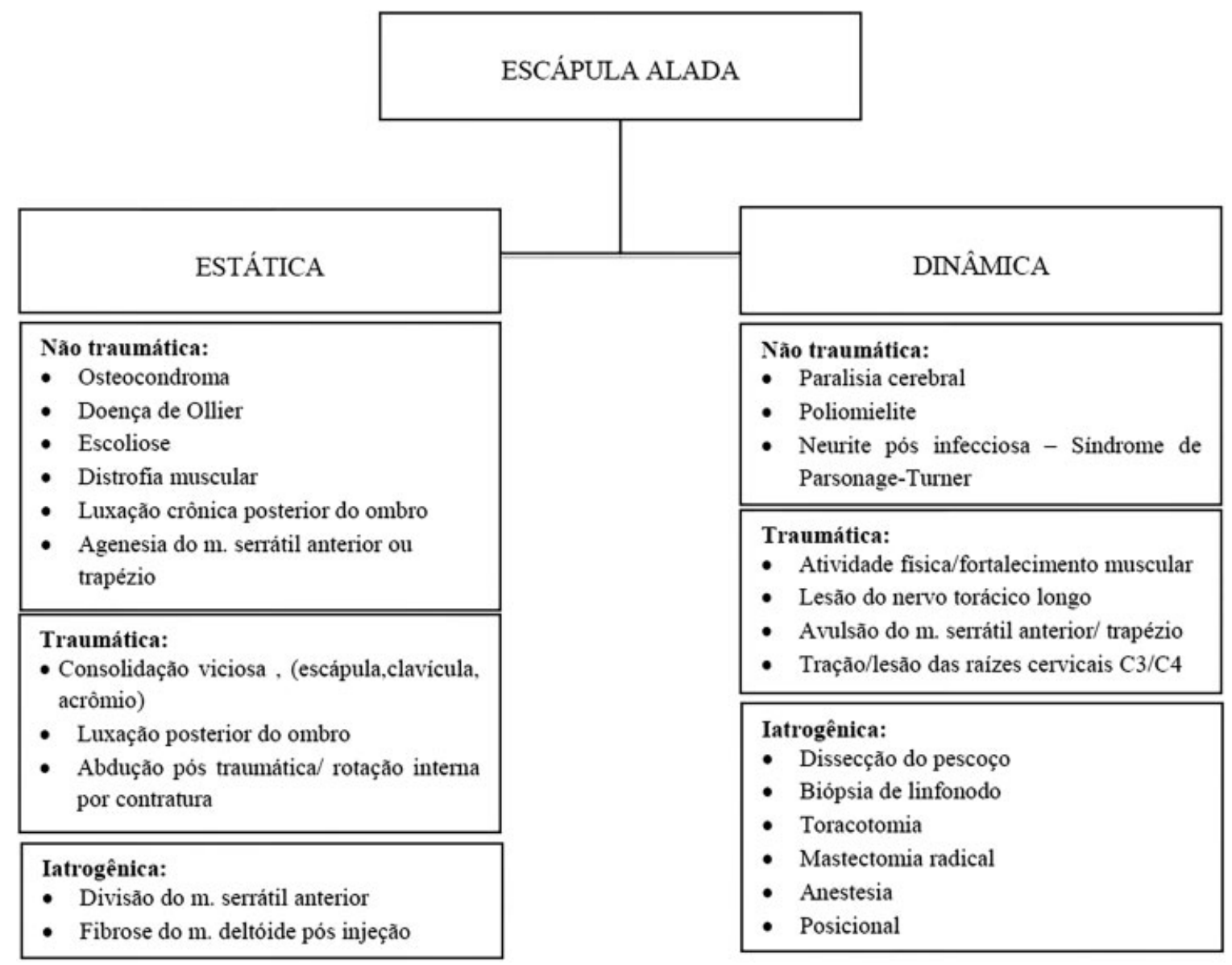

Fig. 4 Diagnósticos diferencias de escápula alada.

inflamatória da mesma. ${ }^{18}$ Orlow, ${ }^{19}$ em 1891 , foi o primeiro a descrevê-la, usando o termo "exostose bursata" para esta localização. No presente estudo, deparou-se com três casos com formação de bursa. Destes, dois apresentavam dor nesta região, o que pode ser um fator de suspeição, pois dentre aqueles que não a apresentavam (57\%), só um referia dor.

Não foi constatada relação entre os dados analisados para fundamentar alguma conclusão pertinente ao tipo, à forma, ao tempo de evolução, à dor, à crepitação e à presença da pseudoescápula alada. As informações são isoladas e sem correlações.

0 tema foi escolhido para enfatizar o organograma para o diagnóstico. As queixas quanto ao osteocondroma são relativamente usuais nos ambulatórios de ortopedia. Entretanto, esta apresentação não é comum e poderá passar desapercebida, perdendo o paciente a chance de ter sua patologia diagnosticada no momento oportuno.

Em se tratando de eventos raros, o tamanho amostral coletado impossibilita a verificação de possíveis relações entre variáveis. Assim, faz-se necessário, em estudos futuros, prolongar o tempo de coleta, ou ampliar o campo de experimento, a fim de recolher mais informações para o estudo em questão.

A principal limitação do presente estudo reside em seu delineamento retrospectivo e observacional, e em não dispor de um grupo controle, porém nossa casuística é maior quando comparada à revisão literária do assunto, na qual as publicações, em sua grande maioria, limitam-se a relatos de caso.

\section{Conclusão}

A escápula alada poderá ter diferentes etiologias. Dentre elas, a presença do osteocondroma retroescapular se faz presente. O conhecimento da anatomia funcional e da semiologia ortopédica, além da correta sistematização da abordagem aos tumores ósseos, deverão ser a base para o diagnóstico diferencial e do tratamento adequado.

Conflitos de Interesses

Os autores declaram não haver conflito de interesses.

\section{Referências}

1 Ermiş MN, Aykut US, Durakbaşa MO, Ozel MS, Bozkuş FS, Karakaş ES. Snapping scapula syndrome caused by subscapular osteochondroma. Eklem Hastalik Cerrahisi 2012;23(01):40-43

2 Unni KK, Inwards CY, Dahlin DC. Dhalin's bone tumors: general aspects and data on 10,165 cases. 6th ed. Philadelphia: Lippincott Williams \& Wilkins; 2010

3 Mohsen MS, Moosa NK, Kumar P. Osteochondroma of the scapula associated with winging and large bursa formation. Med Princ Pract 2006;15(05):387-390

4 Galate JF, Blue JM, Gaines RW. Osteochondroma of the scapula. Mo Med 1995;92(02):95-97 
5 Bovée JV. Multiple osteochondromas. Orphanet J Rare Dis 2008; $3: 3$

6 Dharmadhikari RP. Painful Snapping and Pseudo-winging Scapula due to a large Scapular Osteochondroma. J Orthop Case Rep 2012; 2(02):10-13

7 Ahmed AR, Tan TS, Unni KK, Collins MS, Wenger DE, Sim FH. Secondary chondrosarcoma in osteochondroma: report of 107 patients. Clin Orthop Relat Res 2003;(411):193-206

8 Chillemi C, Franceschini V, Ippolito G, Pasquali R, Diotallevi R, Petrozza V, et al. Osteochondroma as a cause of scapular winging in an adolescent: a case report and review of the literature. J Med Case Reports 2013;7:220

9 Flugstad NA, Sanger JR, Hackbarth DA. Pseudo-winging of the scapula caused by scapular osteochondroma: review of literature and case report. Hand (N Y) 2015;10(02):353-356

10 McWilliams CA. Subscapular exostosis with adventitious bursa. JAMA 1914;LXIII(17):1473-1474https://jamanetwork.com/journals/jama/article-abstract/437705

11 Martin RM, Fish DE. Scapular winging: anatomical review, diagnosis, and treatments. Curr Rev Musculoskelet Med 2008;1(01):1-11
12 Jindal M. Delayed Presentation of Osteochondroma at Superior Angle of Scapula-A Case Report. J Orthop Case Rep 2016;6(03):32-34

13 Walter J, Kiely N, Cool P, Tyrrell P, Quinlivan RM. An unusual case of scapula winging. Neuromuscul Disord 2013;23(03):277-278

14 Orth P, Anagnostakos K, Fritsch E, Kohn D, Madry H. Static winging of the scapula caused by osteochondroma in adults: a case series. J Med Case Reports 2012;6:363

15 Fiddian NJ, King RJ. The winged scapula. Clin Orthop Relat Res 1984;(185):228-236

16 Garrison RC, Unni KK, McLeod RA, Pritchard DJ, Dahlin DC. Chondrosarcoma arising in osteochondroma. Cancer 1982;49 (09):1890-1897

17 Ceberut K, Korkmaz M, Ergin I, Müslehiddinoglu A. Bursa formation with scapular osteochondroma in hereditary multiple exostosis. J Coll Physicians Surg Pak 2013;23(07):512-514

18 Ali AA, Sharma P, Rege R, Seena CR, Rajesh S. Exostosis Bursata Multimodality Imaging Approach. J Clin Diagn Res 2016;10(09): TD03-TD04

19 Orlow LW. Die exostosis bursata und ihreentstehung. Dtsch Z Chir 1891:293-308 